\title{
Co-Rotating Interaction Regions in 2D Hot-Star Wind Models with Line-Driven Instability
}

\author{
Stanley P. Owocki \\ Bartol Research Institute of the University of Delaware, Newark, DE 19716, USA
}

\begin{abstract}
I review simulations of Co-rotating Interaction Regions (CIRs) in linedriven stellar winds. Previous CIR models have been based on a local, Sobolev treatment of the line-force, which effectively suppresses the strong, small-scale instability intrinsic to line-driving. Here I describe a new "3-ray-aligned-grid" method for computing the nonlocal, smooth-source-function line-force in $2 \mathrm{D}$ models that do include this line-driven instability. Preliminary results indicate that key overall features of large-scale CIRs can be quite similar in both Sobolev and non-Sobolev treatments, if the level of instability-generated wind structure is not too great. However, in certain models wherein the unstable self-excitation of wind variability penetrates back to the wind base, the stochastic, small-scale structure can become so dominant that it effectively disrupts any large-scale, CIR pattern.
\end{abstract}

\section{Introduction}

Co-rotating Interaction Regions (CIRs) are the sprial-shaped density compressions that form from the interaction of higher and lower speed streams in the wind from a rotating star. CIRs have long been observed and studied in the solar wind, but Mullan (1984a,b; 1986) was the first to suggest them as a possible mechanism for producing variable absorption features in UV lines formed in the line-driven stellar winds from hot-stars. To be visible as direct variations in line-profiles formed from globally integrated radiative flux, the associated flow structure must be on a relatively large scale, on order the stellar radius; in these highly supersonic winds, this is of order $v / v_{t h} \gg 1$ larger than the Sobolev length $L \equiv v_{t h} /(d v / d r)$, over which the mean flow speed $v$ increases by a ion thermal speed $v_{t h}$. This suggests that the dynamical evolution of large-scale structure might be adequately simulated using the computationally efficient, local, CAK/Sobolev expression for the line force (Castor, Abbott, and Klein 1975; Sobolev 1960), and indeed this has been a key simplification in previous simulations of CIRs in line-driven winds (Cranmer and Owocki 1995, hereafter C096). A recent review (Owocki 1998) has summarized efforts to apply such Sobolev-theory CIR simulations towards modelling various types of line-profile-variability in hot-star winds, including both the classical, slowly evolving Discrete Absorption Components (DACs), and more recently identified Periodic Absorption Modulations (PAMs). 
The central question I wish to address here is: To what degree is the derived CIR structure sensitive to this approximate Sobolev treatment of the line-force? One particularly important question regards the formation of pre-CIR velocity plateaus; CO96 have been suggested these as a possible origin of DACs, but it is still not clear whether they might in fact be just an artifact of the (artificially?) fast inward wave propagation obtained in a Sobolev treatment (cf. Owocki and Rybicki 1986). Another issue regards the role of the strong, intrinsic, small-scale instability of line-driving; this is effectively suppressed in a Sobolev treatment (Owocki and Rybicki 1984), but, if included, it might substantially alter, or even entirely disrupt, any larger-scale CIR structure.

In the next section ( $\$ 2)$ I outline a newly developed, "3-ray-aligned-grid" approach for extending the nonlocal, "Smooth Source Functiorn" (SSF) lineforce method, used in previous 1D instability simulations (Owocki 1991; Owocki and Puls 1996, 1998), into 2D models that can include both largescale CIRs and small-scale instability-generated structure. I then describe (§3) some initial, still preliminary results indicating that key Sobolev-model features (like velocity plateaus) can indeed still form, but only if the overall level of intrinsic variability initiated near the wind base is not too strong. I conclude (§4) with a brief summary discussion.

\section{3-Ray SSF Approach for 2D Instability Simulations}

A key aspect of line-driven-instability simulations regards the computation of the line force. For the instability-generated flow structure at scales near and below the Sobolev length, a local Sobolev approach fails completely. (See, however, Feldmeier 1998.) Instead, one must apply much more computationally expensive, integral forms that take approximate account of the inherently nonlocal scattering character of the radiative transfer for most important driving lines. Owocki and Puls $(1996,1998)$ discuss various levels for approximating this nonlocal force, including the Smooth-Source-Function (SSF) approach that efficiently accounts for key effects (e.g. line-drag; see Lucy 1984) that control the level of instability. The line-force components are obtained from flux-weighted moments of nonlocal escape functions, each of which requires spatial integrations to obtain the optical depth over a wide range of line frequencies, nested within a frequency averaging over the lineprofile. The computational requirements of evaluating such nested, nonlocal force integrations at each time-step of a hydrodynamical model have till now limited simulations of wind structure to just a 1D temporal evolution in radius, effectively suppressing, quite artificially, any lateral variations.

In developing multidimensional instability models that include lateral structure, a central challenge is thus to develop an approach for efficient evaluation of these escape integrals over a suitable collection of directional rays. As an initial approach, I have been experimenting with an approximate 
3-ray SSF method for computing the nonlocal line-force in $2 \mathrm{D}$ wind models in radius $r$ and azimuth $\phi$. At any given grid point, the nonlocal escape probabilities are evaluated along one radial ray, plus two nonradial rays on opposite sides of the radial direction, set always to have a fixed impact parameter $p<R_{*}$ toward the stellar core. A key trick is to choose the radial spacing so that each nonradial ray intersecting a grid point with indices $\{i, j\}$ will also intersect other grid points $\{i \pm n, j \pm n\}$, for integer $n \geq 1$. This avoids the need to carry out a conceptually complex and computationally costly interpolation between a $(p, z)$ ray grid for the radiation transport, and the $(r, \phi)$ grid for the hydrodynamics (cf. Figures $1 \mathrm{a}$ and $1 \mathrm{~b}$ ). For uniform azimuthal spacing $\Delta \phi$, such ray alignment occurs for radial grids satisfying

$$
r_{i}=\frac{p}{\cos \left[i \Delta \phi+\arccos \left(p / R_{*}\right)\right]} .
$$

Figure 1 illustrates the grid and ray alignment for $\Delta \phi=5^{\circ}(=\pi / 36=0.089$ rad) and $p / R_{*}=\sqrt{0.5}$. Through spatial integration along the 3 such rays for each of the $N_{\phi}$ azimuthal zones, one obtains a "6-stream" description (i.e. in 2 directions along each of the 3 rays) for the required nonlocal escape probabilities from each of the $N_{r} \times N_{\phi}$ grid nodes.

The escape along the two nonradial rays provides a rough treatment of the lateral radiation transport. Because these rays are restricted to always impact within the stellar core radius $\left(p<R_{*}\right)$, they are best suited for approximating the direct component of the line-force; but in the crucial wind-acceleration region near the star, they have a substantial azimuthal component, and so also provide a rough approximation of the azimuthal part of the diffuse lineforce, including, for example, the important lateral "line-drag" effect that is predicted to strongly damp small-scale azimuthal velocity variations (Rybicki et al. 1990). As the rays become increasingly radial at larger radii, this capacity to approximate the lateral, diffuse radiation is lost, but the 3 rays still provide a quite accurate representation of the finite-disk form for the direct line-force. A more serious limitation arises from the severe loss of radial resolution at large radii, as demonstrated by the radial/azimuthal grid aspect ratio,

$$
\frac{\Delta r_{i}}{r_{i} \Delta \phi} \approx \sqrt{\left(r_{i} / p\right)^{2}-1}
$$

which increases as $r_{i} / p$ at large radii. This means that small-scale radial structure can be relatively well-resolved in the inner wind, but then becomes strongly damped by grid-averaging in the outer wind.

¿From the frequency-dependent optical depth along the 3 rays, one can obtain the corresponding integral escape probabilities in the outward and inward directions, and then from the flux moment of these, compute the direct and diffuse contributions to the line-force. (See eqs. 65 and 67 of Owocki and Puls 1996.) In addition to the radial line-force that drives the wind outflow, there is, in general, a nonzero azimuthal force component as well. In a rotating wind, this can arise even when the wind itself is axially symmetric, because 
a.

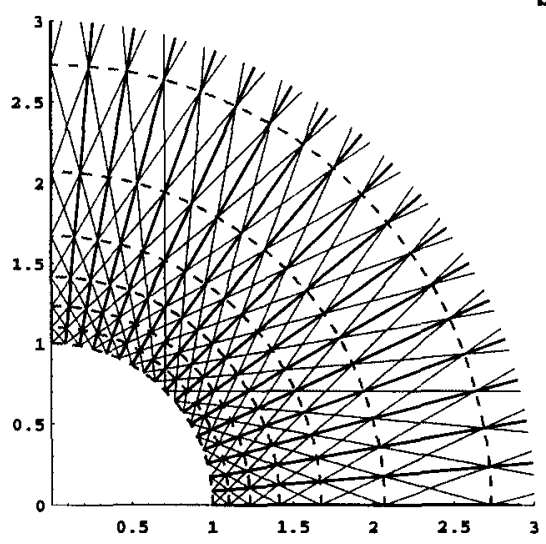

b.



Fig. 1. a.) Illustration of 3-rays with $p / R_{*}=0, \pm \sqrt{0.5}$ spaced azimuthally every $\Delta \phi=5^{\circ}$, together with the radial grid spacing (dashed arcs) that aligns the rays to intersect multiple $(r, \phi)$ grid nodes; this allows very efficient evaluation of the ray escape integrals needed for computation of the SSF line-force in 2D wind models. b.) Contrasting the much greater complexity of a nonaligned ray-grid with about the same total number of rays, now with a coarser azimuthal resolution $\Delta \phi=15^{\circ}$, but spaced every $\Delta p=\Delta r=R_{*} / 2$ over the full model range $0<p<3 R_{*}$; the chaotic, nonaligned ray intersection means that extensive interpolation would be needed both to carry out $(p, z)$ ray integrations, and then to apply these toward computing the line-force on the $(r, \phi)$ hydro grid.

the different velocity gradients in the prograde and retrograde directions still imply a corresponding asymmetry in the escape probabilities (Grinin 1978). The resulting torque can lead to a moderate $(\sim 20-30 \%)$ spin-down of the wind rotation. (See, e.g., figure 3 in Owocki et al. 1998.)

\section{Preliminary Results of 3-Ray SSF Models of CIRs}

Following the general approach introduced by CO96, CIRs and other azimuthal wind variations are induced here by enhancing the wind driving from an isolated, bright spot on the rotating stellar surface. However, whereas CO96 took the line-force to be strictly radial, with a fixed enhancement factor given by the relative proximity to the spot, the simulations here compute both radial and azimuthal components of the line-force directly from the 3-ray quadrature (with the surface brightness contribution to each ray appropriately averaged to account for the larger angle range visible from greater heights). In addition to the overall spindown effect noted above, there is now also an azimuthal line-force contribution directed away from the bright spot. The models here invoke a spot with amplitude and width similar to the 

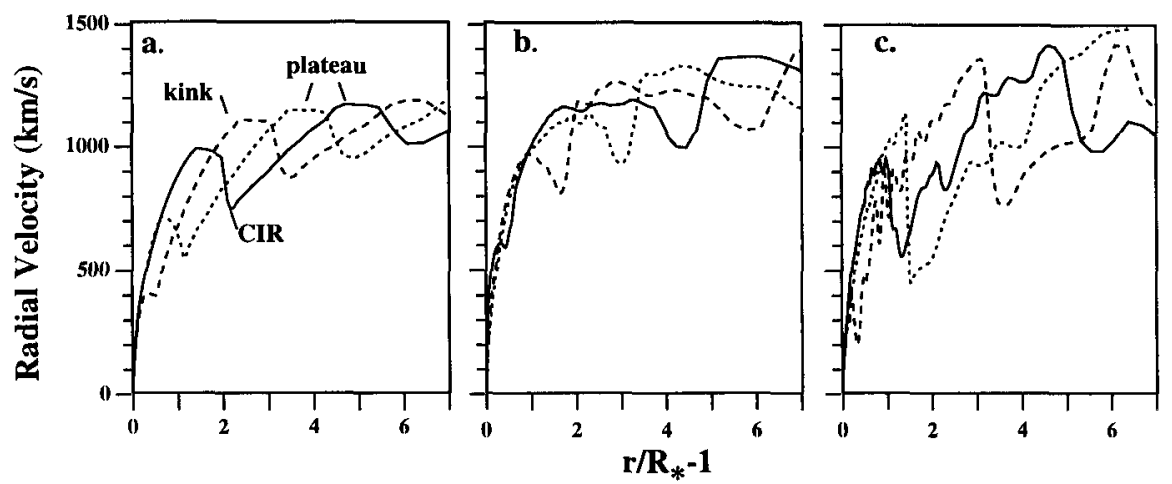

Fig. 2. Radial variation of radial velocity along selected azimuthal angles in spot-induced CIR models, with line-force treatments based on (a.) the local, CAK/Sobolev method, and the 3-ray SSF method with (b.) $p / R_{*}=\sqrt{0.5}$ and no azimuthal forces, and (c.) $p / R_{*}=\sqrt{0.8}$, including azimuthal forces.

"standard bright spot model" of CO96, with, however, a horizontal periodicity of just $45^{\circ}$ (vs. $180^{\circ}$ ), simply to reduce computational expense. This azimuthal range is divided into $N_{\phi}=157$ azimuthal zones of equal width, $\Delta \phi=0.005 \mathrm{rad}=0.287^{\circ}$.

Below I compare results of a CAK/Sobolev CIR simulation ("Model A") with two representative SSF models (" $B$ " and " $C$ ") distinguished by the relative weighting of the lateral transport. Model $B$ neglects the azimuthal force, and uses a moderate impact parameter $p / R_{*}=\sqrt{0.5}$. Model C includes the azimuthal force, and uses a larger impact parameter $p / R_{*}=\sqrt{0.8}$ that gives greater weight to the lateral radiation. The lower boundary $r=R_{*}$ begins with horizontal/azimuthal grid aspect ratios of 1 and $1 / 2$ for models A and B, which require respectively $N_{r}=200$ and $N_{r}=141$ radial zones to reach the assumed maximum model radius $R_{m a x} \approx 8 R_{*}$. As in CO96, the stellar and wind parameters are chosen to represent a standard O-supergiant (e.g. $\zeta$ Pup) with rotation speed, $v_{\text {rot }}=200 \mathrm{~km} / \mathrm{s}$.

Figure 2 compares results for the radial variation of radial velocity along selected aziumthal angles in various spot-induced CIR models. Panel (a) shows the characteristic structure of the CAK/Sobolev model A, with a nearly flat velocity plateaus upwind from the velocity minima that signify the dense CIR, extending back to a velocity gradient discontinuity, or "kink", that marks the connection to the unperturbed, outward-accelerating wind. As discussed in CO96, this weak, kink discontinuity propagates inward (relative to the wind outflow) at a characteristic speed $c_{-} \approx-v$ that is nearly as fast as the local outflow speed $v$, yielding a quite slow net outward propagation in 


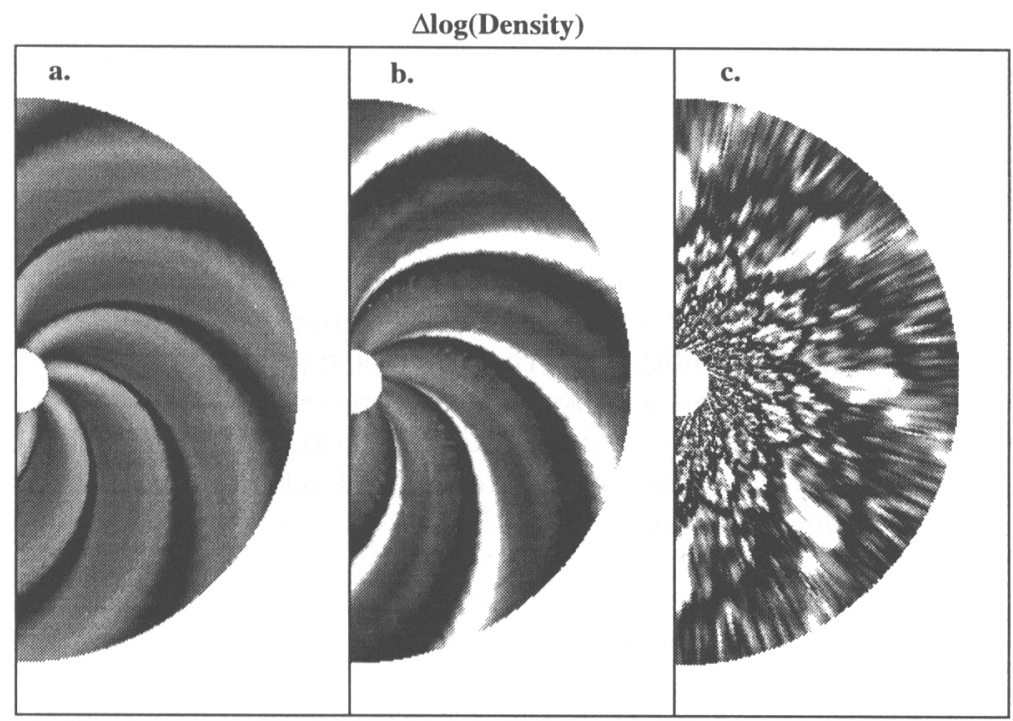

Fig. 3. 2D grey-scale representation of the density variation in the same three CIR models depicted in figure 2 .

the fixed stellar frame. The slowly evolving flat velocity plateau that forms between the kink and the CIR moreover gives rise to an overall line-profile variability that has many of the observed characteristics of slowly evolving DACs (CO96).

Figure $2 \mathrm{~b}$ shows that the corresponding 3-ray SSF model $\mathrm{B}$ has a roughly similar overall velocity variation, including extended velocity plateaus. In fact, apart from some moderate waviness in the velocity, there is little of the small-scale structure expected from the line-driven instability. By contrast, analogous $1 \mathrm{D}$ models typically show extensive, intrinsic wind structure within about $R_{*} / 2$ from the stellar surface, even without any explicit perturbations. As discussed by Owocki and Puls (1998), this is apparently the consequence of backscattered radiation from the structured outer wind "self-seeding" perturbations at the wind base, which are then amplified by the strong instability growth. The lack of such self-seeded structure here is likely an artifact of the rapidly increasing radial grid spacing, which smooths out small-scale variations in the outer wind, effectively breaking the self-excitation cycle.

Remarkably, figure 2c shows that the 3-ray SSF model C with $g_{\phi} \neq 0$ and just a slightly different grid parameterization has a dramatically different velocity structure, dominated by small-scale instability variation throughout the wind, including right down to the wind base. This effectively destroys any large-scale features, including the extended velocity plateaus. 
Figures 3 a-c show 2D grey-scale plots of the corresponding density variation, measured logarthmically relative to the azimuthal average. The clear spiral CIR pattern of the CAK/Sobolev spot model in panel (a) is still quite apparent in the somewhat more variable 3-ray SSF model in panel (b); but again this large-scale CIR pattern is almost completely disrupted by the extensive small-scale structure arising in the intrinsically unstable case in panel (c).

Other simulation models we have run indicate that both the inclusion of the azimuthal force and the larger lateral ray impact parameter tend to increase the intrinsic variability, with the latter being the stronger effect. The reasons for this are still unclear, and indeed run contrary with the expectation from 3D linear stability analyses (Rybicki et al. 1990) that indicate the diffuse lateral line-force should dampen azimuthal velocity variations, and so presumably stabilize the flow.

\section{Summary Discussion}

I caution that the results presented here are still very preliminary. Because the reasons for the marked difference in the level of intrinsic, small-scale variability are still unclear, it is not yet possible to say which of these two extreme scenarios is more likely to represent conditions in actual stellar winds. Nonetheless these results do point to two tentative new conclusions. 1.) The formation of pre-CIR velocity plateaus is not strictly an artifact of using a CAK/Sobolev form for the line-force. 2.) However, such plateaus, and indeed the entire large-scale CIR structure, can be completely disrupted if the level of small-scale, intrinsic instability becomes too strong. In this sense, the 3-ray SSF method introduced here has, despite its still very approximate nature, provided some intriguing first insights into the dynamical processes that control the multidimensional and multiscale structure that likely exists in the highly unstable and highly variable line-driven stellar winds from hot, luminous stars.

Acknowledgements: This work was supported in part by NASA grant NAGW-2624. The computations were carried out using an institutional allocation of supercomputer time from the San Diego Supercomputer Center. I acknowledge numerous helpful discussions with S. Cranmer, A. Feldmeier, A. Fullerton, K. Gayley, and J. Puls.

\section{References}

Castor, J. I., Abbott, D. C., \& Klein, R. I. 1975, Ap. J., 195, 157 (CAK)

Cranmer, S. R., \& Owocki, S. P. 1996, ApJ, 462, 469 (CO96)

Feldmeier, A. 1998, Ap. J., in press

Grinin, A., 1978, Sov. Astr. 14, 113 
Lucy, L. B. 1984, Ap. J. 284, 351

Mullan, D. J. 1984a, Ap. J., 283, 303

Mullan, D. J. 1984b, Ap. J., 284, 769

Mullan, D. J. 1986, Astron. \& Ap., 165, 157

Owocki, S. P., 1991, Stellar Atmospheres: Beyond Classical Models, L. Crivellari, I. Hubeny, D. G. Hummer, eds. (Kluwer: Dordrecht), 235

Owocki, S.P. 1998, Cyclical Variability in Stellar Winds, L. Kaper and A. Fullerton, eds., ESO Astrophysics Symposia Series, (Springer: Heidelberg), 325

Owocki, S. P., Castor, J. I., \& Rybicki, G. B., 1988, Ap. J. 335, 914 (OCR)

Owocki, S.P., Cranmer, S.R., Gayley, K.G., 1998, in proceedings of Workshop on

B[e] Stars, held in Paris, France, June, 1997, A. Hubert, ed., Kluwer, in press

Owocki, S. P., \& Puls, J. 1996, Ap. J. 462, 894

Owocki, S. P., \& Puls, J. 1998, Ap. J, in press

Owocki, S. P., \& Rybicki, G. B. 1985, Ap. J., 199, 365

Owocki, S. P., \& Rybicki, G. B. 1986, Ap. J., 209, 127

Rybicki, G. B., Owocki, S. P., \& Castor, J. I. 1990, Ap. J., 349, 274

Sobolev, V. V. 1960, Moving Envelopes of Stars (Cambridge: Harvard University Press)

\section{Discussion}

T. Rivinius: Can the spin-down in the wind account for detached Be star disks?

S. Owocki: No, the effect only works on outwardly accelerating flows. There is no effect for material in a stable Keplerian disk.

A. Feldmeier: You mentioned that an inward propagating perturbation is only expected when the diffuse force is included in the calculation. Would you expect that the inward propagating velocity plateau in your CIR model disappears when using the pure absorption line force?

S. Owocki: In principle, I would expect this to be the case, because the pure absorption model does not allow inward propagation of information faster than the sound speed. However, because of the very strong instability of the pure-absorption case, including base variability associated with the degeneracy of the overall wind solution (see Poe et al., 1990, ApJ 358, 199), it is difficult in practice to test this notion, because all large-scale features like velocity plateaus simply become totally disrupted by small-scale structure.

N. Langer: Is the spin-down effect for hot star winds you found the end of wind-compressed disks or even wind compression per se?

S. Owocki: No, but the discovery of spin-down is what led me to investigate WCD models with non-radial forces. But it turns out that the latitudinal force is much more important for inhibiting wind compression. In fact, in early phases of P. Petrenz's independent simulation he accidently left out the latitudinal force, while including the spin-down effect, and found such models show little reduction in WCD. With all non-radial forces included, 
our independent codes now agree very well on WCD inhibition.

J. Cassinelli: When you included the most sophisticated radiation treatment, you lost the CIRs. Then you went back to the less sophisticated treatment and found the decrease of wind angular momentum. So, we might be left with the impression that when you again do the sophisticated model you will lose the angular momentum decrease and perhaps also the inhibition.

S. Owocki: Sorry, this was mostly a poor choice in the order of presentation. The spin-down effect was discovered using the "most sophisticated" non-local treatment, but its basic cause can be understood in local CAK/Sobolev models. However, non-local effects actually amplify the effect near the sonic point. Furthermore, it is not quite right that CIRs are necessarily lost in the "most sophisticated models". If instability is not initiated too close to the wind base, CIRs are quite distinct even in the non-local force models. Finally, I agree that the effect of instability on WCD inhibition needs to be investigated.

G. Mellema: Would the shape of the PAMs depend on the pitch angle of the spiral pattern? Could we then use this to derive wind parameters?

S. Owocki: That is an interesting possibility that can in principle be investigated with $3-\mathrm{D}$ spot/CIR models. These would be quite doable with modern computers.

F. Vakili: Do you have any idea about the origin of the asymmetry we detect with our interferometric observations of $\mathrm{P}$ Cygni in $\mathrm{H} \alpha$, noting that this asymmetry is found very close to the central star?

S. Owocki: Whenever the mass loss varies with latitude, whether increasing toward the pole or equator, it seems emission from near the star will be asymmetric from any perspective that is not looking directly pole-on.

H. Henrichs: May I confront your wonderful theory with observations of $\xi$ Per (showing Si IV DACs)? How do you define PAMS in this plot?

S. Owocki: I would concentrate on whether there is a clear increase in absorption or a modulation between increased and decreased absorption. The former suggests DACS; the latter PAMs. At first glance, it seems your example might be a case where PAMs in the inner wind evolve outward into DACs.

J. de Jong: $\xi$ Per does show phase bowing in Si IV and the time cross-sections are quite sinusoidal. This seems to point to PAMs rather than DACs. 